\title{
Angiographic and Clinical Features of Noninvoluting Congenital Hemangiomas
}

\author{
(D)A. Patel, DR. De Leacy, and (D) A. Berenstein
}

\begin{abstract}
SUMMARY: While infantile hemangiomas are very common, congenital hemangiomas are rare and less understood. Congenital hemangiomas are present at birth. They fall into 2 major categories: rapidly involuting congenital hemangioma and noninvoluting congenital hemangioma. Noninvoluting congenital hemangioma is the rarer of the 2 entities. If not recognized and treated appropriately, noninvoluting congenital hemangioma can lead to considerable morbidity. There is a paucity of literature regarding noninvoluting congenital hemangiomas. In this article, we will outline our experience with this condition, focusing on clinical and angiographic features. There is a distinct angiographic appearance of noninvoluting congenital hemangiomas involving an arterial-capillary web, a dense tumor blush with identifiable feeding arteries, no arteriovenous shunting, and variably present draining veins. Our experience with endovascular embolization and direct percutaneous treatment is the largest for this entity to date. Endovascular embolization and/or direct percutaneous sclerotherapy of this lesion may obviate subsequent surgical resection.
\end{abstract}

ABBREVIATION: $\mathrm{NICH}=$ noninvoluting congenital hemangioma

$\mathrm{H}$ emangiomas are vascular tumors that enlarge through proliferation of endothelial cells. Infantile hemangiomas are common lesions, occurring in up to $10 \%$ of infants and young children. ${ }^{1}$ These tumors generally appear in the first 6 weeks of life and subsequently undergo a spontaneous involution phase. Digital subtraction angiography, if performed, will demonstrate dilated feeding arteries, organized arterial angioarchitecture, a dense parenchymal blush, and drainage into dilated draining veins. ${ }^{1}$ Infantile hemangiomas may demonstrate rapid venous filling, indicating arteriovenous shunting.

Congenital hemangiomas are much rarer lesions. Physicians caring for pediatric patients with hemangiomas have long noted that infants would occasionally present with congenital hemangiomas. ${ }^{2}$ These hemangiomas undergo their proliferative phase in utero. Therefore, while they can still grow proportionally as the

Received November 16, 2018; accepted after revision March 2, 2019.

From the Department of Radiology (A.P.), New York Presbyterian-Weill Cornell Medical Center, New York, New York; and Cerebrovascular Center (R.D.L., A.B.), Icahn School of Medicine at Mount Sinai, New York, New York.

Previously presented at: American Society of Neuroradiology Annual Meeting and the Foundation of the ASNR Symposium, June 2-7, 2018;

Vancouver, British Columbia, Canada.

Please address correspondence to Anant Patel, MD, Department of Radiology, New York Presbyterian-Weill Cornell Medical Center, 520 E 70th Street, Starr 8a, New York, New York 10021; e-mail: arp9071@med.cornell.edu; @AnantPatelMD

$\equiv$ Indicates article with on-line table.

http://dx.doi.org/10.3174/ajnr.A6044

patient ages, these hemangiomas will not demonstrate accelerated or disproportionate postnatal growth. The pathologic characteristic distinguishing these lesions from the more common infantile hemangioma is the lack of expression of glucose transporter isoform 1 (GLUT1). ${ }^{3}$

There are 2 types of congenital hemangiomas: rapidly involuting congenital hemangiomas and noninvoluting congenital hemangiomas (NICHs). These lesions rarely coexist with the more common infantile hemangiomas and are usually solitary. As the name implies, rapidly involuting congenital hemangioma lesions undergo a rapid involuting phase, and most cases demonstrate complete involution within the first 6-14 months of life. ${ }^{4}$ Management of these lesions is straightforward if the patient presents typically. Observation is, in general, the initial approach. Lesions that do not involute may need biopsy to exclude a tumor such as rhabdomyosarcoma or congenital fibrosarcoma.

Noninvoluting congenital hemangiomas are even rarer than rapidly involuting congenital hemangioma lesions. Most lesions occur in the head and neck, trunk, or limbs. These lesions tend to be plaquelike, pink-to-purple with telangiectasia on the surface. They are often warm to touch. Noninvoluting congenital hemangiomas stain negative for GLUT1, and a characteristic hobnailed appearance is often seen on pathology. As their name implies, these lesions will never disappear or involute. Imaging often demonstrates hyperintensity on T2-weighted images with flow voids similar to those in infantile hemangiomas. A limited description of the angiographic features of these lesions and endovascular 

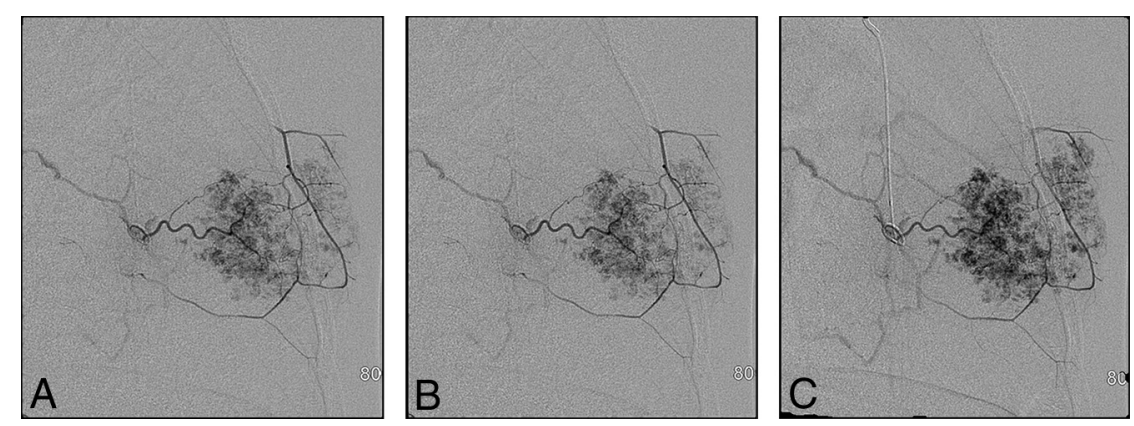

FIG 1. Left internal maxillary artery injection of a NICH of the left anterior chest wall. The lesion was initially treated with QuadraSphere Microspheres; the treatment was not successful. Subsequent treatment with an ethanol injection led to involution.
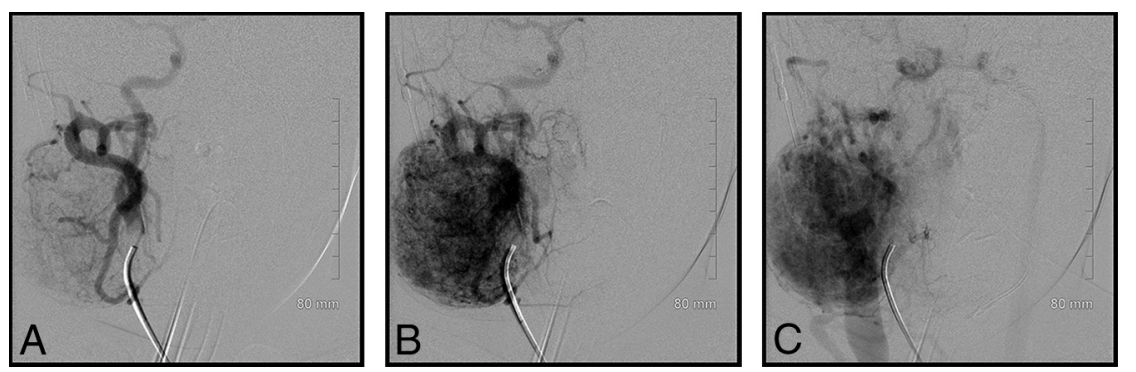

FIG 2. Right external carotid artery injection of a right facial $\mathrm{NICH}$. Again, there is a discrete angiographic appearance involving an arteriocapillary web, no arteriovenous shunting, and identifiable draining veins. This lesion was treated successfully with polyvinyl alcohol particles.

approaches for treating them is available. ${ }^{5}$ The literature focuses on resection as the mainstay of treatment. Previous, limited reports of endovascular embolization are only in the context of a preoperative setting to control bleeding in larger lesions during subsequent resection. Here, we report our experience with this entity and demonstrate that endovascular embolization and/or direct percutaneous sclerotherapy can be an effective treatment strategy in well-selected cases.

\section{Case Series}

Case Selection. Using Mount Sinai institutional review boardapproved protocol, all cases of noninvoluting congenital hemangiomas from 1990 to present were identified for this retrospective study. Ten patients were identified from the January 1990 to May 2017. All cases were patients who had been referred for endovascular embolization and/or direct percutaneous sclerotherapy. All patients were treated. Indications for treating NICH included cosmetic deformity, location not suitable for gross resection, lesions previously treated but not regressing, or the need to shrink the size of the lesion so that it could be amenable to a subsequent resection.

Lesion location, clinical scenario, patient age, angiographic features, embolization technique, postembolization angiography, need for multiple treatments (if applicable), complications, and long-term clinical follow-up were recorded. Comparison with any prior or subsequent imaging after angiography was performed to document stability. When possible, we accessed pathology reports confirming GLUT1 negativity in these patients with $\mathrm{NICH}$.

\section{Angiography and Endovascular Embolization}

For patients undergoing angiography and embolization, the right common femoral artery was accessed. A 4F sheath was placed. A 4F Berenstein catheter (Boston Scientific, Fremont, California) and/or Prowler 10 microcatheter (Codman \& Shurtleff, Raynham, Massachusetts) was used to superselect the vessel of interest. Superselective injections with the microcatheter along the arcade of the vascular lesion would be performed to confirm an angiographic appearance of the NICH.

Embolization was performed once distal catheterization was achieved and it was determined that the patient's lesion was suitable for treatment. In most cases, we used $n$-BCA. Specifically, we generally used $0.7 \mathrm{~mL}$ of $25 \% n$-BCA ( $1 \mathrm{~mL}$ of $n$-BCA mixed with $3 \mathrm{~mL}$ of Ethiodol [Andre Guerbet, Melville, New York]) for injection. The relatively small volume of $n$-BCA reflects the relative overall volumes of these lesions (most patients were small children), limited number of pedicles in most lesions, and the primary objective of the $n$-BCA often being to slow down the flow in the main pedicle.

\section{Direct Percutaneous Sclerotherapy}

Patients presenting with very large hemangiomas undergoing direct percutaneous sclerotherapy were also reviewed. This technique was used more frequently across time as the practice of our primary operator evolved. Under direct fluoroscopic monitoring, direct percutaneous puncture of the lesion of interest at multiple locations was performed. Following establishment of blood flow, a small amount of contrast material was injected to confirm the intravascular location of our angiocatheter. Subsequently, we would sclerose the hemangioma with progressive small injections of $98 \%$ ethanol. Given the nature of the lesions, we would inject either in the central portion of the mass or in separate compartments to decrease the overall size. Many cases indeed used multiple, separate puncture sites in which ethanol was injected followed by $\sim 5 \mathrm{~mL}$ of Avitene microfibrillar collagen hemostat suspension (Bard Davol, Warwick, Rhode Island) for hemostasis.

\section{Outcomes}

We identified 10 patients who underwent 25 total treatments. The On-line Table summarizes the key clinical and angiographic features of our patients. Most of our patients presented between 2 and 8 years of age. One patient underwent treatment at 44 years of age. Seven patients were female, and 3 were male. Five patients were treated with endovascular embolization, 2 patients were treated with percutaneous sclerotherapy, and 3 patients were 
treated with a combination of the 2 approaches as the practice of our primary operator evolved.

Patients were seen in clinic between 1 and 2 months after treatment and at 12 months. As shown in the On-line Table, most patients required between 1 and 2 treatments to achieve 12 months without lesion recurrence or progression. One of our patients did require a total of 12 treatments (in addition to 3 prior endovascular treatments at a peer institution) before surgical resection. Another patient required 2 treatments before surgical resection. The remaining 8 patients did not need an operation.
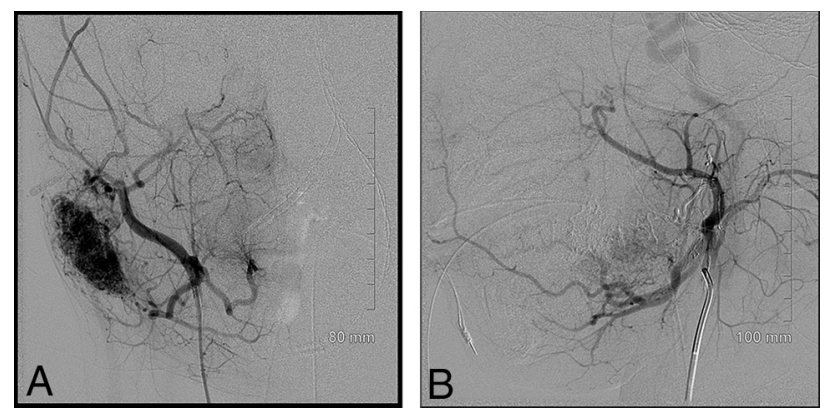

FIG 3. Pre- and postembolization images of a right parotid $\mathrm{NICH}$ treated with $n$-BCA. There were 5 vascular pedicles seen on angiography, and each was injected with $25 \% n$-BCA $(0.15-0.45 \mathrm{~mL}$ per pedicle).

\section{DISCUSSION}

Our cases highlight several important points: noninvoluting congenital hemangiomas have the same angiographic appearance throughout the body. Figure 1 of a case involving an NICH in the anterior chest wall shows the same features as a facial NICH, including a discrete arterial-capillary web, homogeneous tumor blush, no arteriovenous shunting, and draining veins. This lesion was initially treated with QuadraSphere Microspheres (Merit Medical, South Jordan, Utah) (and did not significantly involute). The lesion was subsequently successfully treated with ethanol. Figure 2 highlights a right facial NICH with similar angiographic features. This lesion was successfully treated with polyvinyl alcohol particles, 45-120 $\mu \mathrm{m}$. Markedly enlarged draining veins are noted in this example.

Contrary to previous reports, our experience shows that in some cases, endovascular embolization or direct percutaneous sclerotherapy can obviate surgical resection. The largest previous case series on NICH identified 53 patients across 3 large vascular anomaly centers. ${ }^{5}$ However, this study had a limited description of angiographic features, and only 7 of 53 patients underwent embolization, which was performed explicitly to possibly decrease bleeding in a subsequent resection. This article goes on to say that "embolization is unnecessary." Two of our cases showed that preprocedural embolization can considerably decrease intraoperative bleeding. More important, 6 of our cases suggest that effective endovascular embolization and/or direct sclerotherapy can entirely obviate a resection.
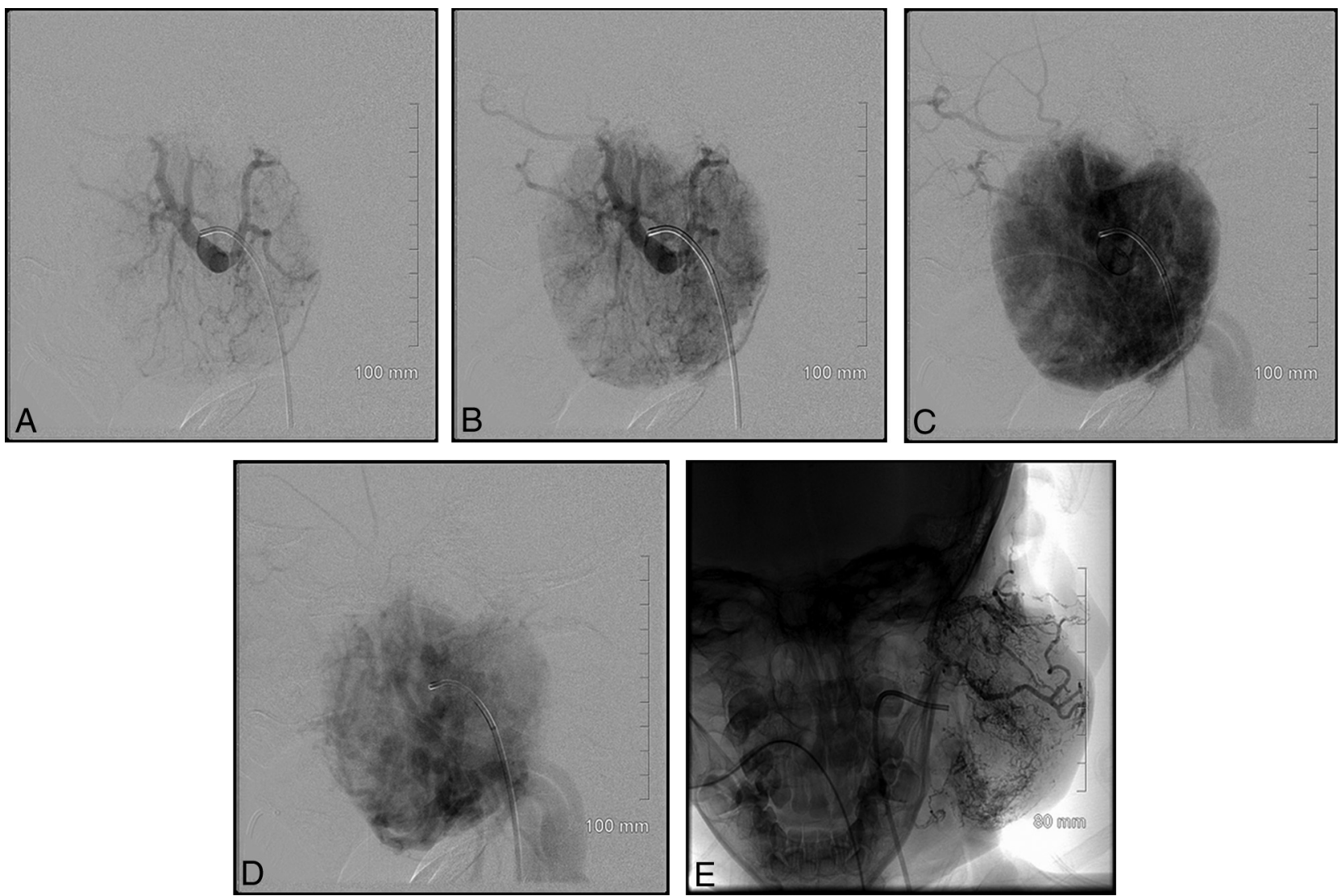

FIG 4. An incredibly vascular left parotid lesion. This lesion was treated with both endovascular embolization and direct percutaneous sclerotherapy. Postembolization image $(E)$ from this case shows a markedly reduced vascular supply. 
Indeed, we have seen that endovascular embolization and/or direct sclerotherapy can effectively decrease the vascular supply, lead to involution, and, in appropriately selected cases, be a definitive treatment.

Another important observation from our experience is that both endovascular embolization and direct percutaneous sclerotherapy are effective. Figure 3 highlights a right parotid NICH in a 4-year-old patient. The lesion was not regressing. A dense tumor stain was observable as well as 5 discrete vascular pedicles feeding the lesion. The lesion was successfully treated with $n$-BCA.

Direct percutaneous sclerotherapy is another technique that can be used. Our group's experience is that this technique works well in select cases. Moreover, use of this procedure increased with time, mirroring the evolution of our primary operator's practice. Figure 4 highlights a case using both endovascular embolization and direct percutaneous sclerotherapy with Avitene. This was an extremely vascular tumor, and the combination of both techniques led us to achieve occlusion of approximately $80 \%$ of the tumor.

Our study has some limitations. Our case series includes only 10 patients. However, this mainly underscores the rare incidence of this condition. Only 2 patients had a biopsy before our intervention, which showed GLUT1 negativity, confirming NICH. The remaining patients did not have a preprocedural biopsy but were noted to have a clinical scenario reliably demonstrating an $\mathrm{NICH}$ lesion. Moreover, because a subsequent operation was not necessary, no sample of tissue was sent for GLUT1 staining.

Five of our patients needed only 1 treatment. One patient was noted to have undergone 12 embolizations with us, many of which were after surgical resection. Additionally, this patient had 3 prior embolizations at Johns Hopkins.

\section{CONCLUSIONS}

Our study is the largest case series of embolization and/or direct sclerotherapy for NICH and the most thorough evaluation of angiographic features associated with these lesions. NICHs have distinguishing angiographic and clinical features. Endovascular or direct percutaneous embolization or both are effective treatment strategies that may obviate surgical resection.

Disclosures: Reade De Leacy—UNRELATED: Board Membership: Asahi Intecc, Comments: research grant*; Consultancy: Siemens, Penumbra. Alejandro BerensteinRELATED: Grant: MicroVention; Consulting Fee or Honorarium: MicroVention, Mivi Neuroscience, Scientia Vascular, Endostream Medical LTD, Bendit Technologies, Magneto Thrombectomy Solutions; UNRELATED: Royalties: AngioDynamics; Stock/Stock Options: Mivi Neuroscience, Endostream Medical LTD, Scientia Vascular, Bendit Technologies, Magneto Thrombectomy Solutions. *Money paid to the institution.

\section{REFERENCES}

1. Song JK, Niimi Y, Berenstein A. Endovascular treatment of hemangiomas. Neuroimaging Clin N Am 2007;17:165-73 CrossRef Medline

2. Krol A, MacArthur CJ. Congenital hemangiomas: rapidly involuting and noninvoluting congenital hemangiomas. Arch Facial Plast Surg 2005;7:307-11 CrossRef Medline

3. North PE, Warner M, Mizeracki A, et al. GLUT1: a newly discovered immunohistochemical marker for juvenile hemangiomas. Hum Pathol 2000;31:11-22 CrossRef Medline

4. Mulliken JB, Enjoiras O. Congenital hemangiomas and infantile hemangioma: missing links. J Am Acad Dermatol 2004;50:875-82 CrossRef Medline

5. Enjolras O, Mulliken JB, Boon LM, et al. Noninvoluting congenital hemangioma: a rare cutaneous vascular anomaly. Plast Reconstr Surg 2001;107:1647-54 CrossRef Medline 